\title{
A Metabolomic Study of Cervical Dystonia
}

Chang Liu, $\mathrm{MPH}^{1}$, Laura Scorr, $\mathrm{MD}^{2}$, Gamze Kilic-Berkmen, $\mathrm{PhD}^{2}$, Adam Cotton, $\mathrm{BA}^{2}$, Stewart A. Factor, $\mathrm{DO}^{2}$, Alan Freeman, $\mathrm{MD}^{2}$, ViLinh Tran, $\mathrm{MS}^{3}$, Ken Liu, $\mathrm{PhD}^{3}$, Karan Uppal, $\mathrm{PhD}^{4}$, Dean Jones, $\mathrm{PhD}^{4}$, H. A. Jinnah, MD $\mathrm{PhD}^{2,5}$, Yan V. Sun, $\mathrm{PhD}^{1,6}$

${ }^{1}$ Department of Epidemiology, Emory University Rollins School of Public Health, Atlanta, Georgia, USA

2 Jean and Paul Amos Parkinson's Disease and Movement Disorder Clinic Department of Neurology, Emory University School of Medicine, Atlanta, Georgia, USA

${ }^{3}$ Clinical Biomarkers Laboratory, Department of Pulmonary, Allergy, Critical Care and Sleep Medicine, Emory University School of Medicine, Atlanta, Georgia, USA

${ }^{4}$ Department of Pulmonary, Allergy, Critical Care and Sleep Medicine, Emory University School of Medicine, Atlanta, Georgia, USA

${ }^{5}$ Department of Human Genetics, Emory University School of Medicine, Atlanta, Georgia, USA

${ }^{6}$ Department of Biomedical Informatics, Emory University School of Medicine, Atlanta, Georgia, USA

\section{Corresponding Author:}

Yan V. Sun, PhD

Department of Epidemiology, Rollins School of Public Health

Emory University

1518 Clifton Rd. NE, Atlanta, Georgia 30322

Tel: 404-727-9090

Fax: 404-727-8737

Email: yan.v.sun@emory.edu

Word Count: 1,709 (Abstract 166)

Running Title: Cervical Dystonia Metabolomics

Keywords: Cervical dystonia, metabolomics, torticollis, MWAS 


\begin{abstract}
Background: Cervical dystonia is the most common of the adult-onset focal dystonias. Most cases are idiopathic. The current view is that cervical dystonia may be caused by some combination of genetic and environmental factors. Genetic contributions have been studied extensively, but there are few studies of other factors.
\end{abstract}

Objective: To conduct an exploratory metabolomics analysis of cervical dystonia to identify potentially abnormal metabolites or altered biological pathways.

Methods: Plasma samples from 100 cases with idiopathic cervical dystonia and 100 controls were compared using liquid chromatography coupled with mass spectrometry-based metabolomics.

Results: A total of 7,346 metabolic features remained after quality control, and 289 demonstrated significant differences between cases and controls, depending on statistical criteria chosen. Pathway analysis revealed 9 biological processes to be significantly associated at $\mathrm{p}<0.05,5$ pathways were related to carbohydrate metabolism, 3 pathways were related to lipid metabolism.

Conclusion: This is the first large scale metabolomics study for any type of dystonia. The results may provide potential novel insights into the biology of cervical dystonia. 


\section{Introduction}

Cervical dystonia (CD) is characterized by excessive involuntary contractions of muscles of the neck and shoulder leading to abnormal head postures, involuntary movements including tremor, and pain [1]. It is considered to be a rare disorder with an overall incidence of 1.2 per 100,000 person years [2], and crude prevalence of 29.5 per 100,000 population [3]. It typically begins in adults between 40-60 years of age, with females being affected more often than males [4]. For the majority of cases, a cause cannot be found, even after extensive diagnostic testing. Much of the recent research regarding potential causes has focused on genetics, as $\sim 15 \%$ of patients with CD have a family member affected with dystonia [4]. However, the genes currently known to cause dystonia account for no more than $4 \%$ of all cases [1]. These observations suggest that other, as yet undiscovered, genes may play a role.

Non-genetic factors may also have a place in pathogenesis. Rare cases of CD may be caused by focal lesions of the nervous system [5], exposure to medications such as neuroleptics $[6,7]$, infection [8], or autoimmune mechanisms [9]. A few small studies have methodically addressed other potential environmental contributions. A survey study of 184 cases of all types of idiopathic dystonia in Australia suggested cigarette smoking to be a risk factor [10]. A case control study of 67 cases of CD suggested a role for trauma to the head and neck [11].

The metabolome is the global collection of all small molecules in the body. It reflects the combined systemic effects of genetics, lifestyle, environmental exposure and secondary biological responses. Metabolomics, or the study of global metabolism, is an emerging discipline that has the potential to transform the study of the mechanism of disease development. Highresolution chemical profiling involves the use of liquid chromatography to separate analytes and high-resolution mass spectrometry to accurately measure mass and abundance $[12,13]$. To our 
knowledge, there are no published metabolomic studies for any of the common adult-onset focal dystonia. The current study describes results of a blood-based metabolomics study of 100 subjects with CD and 100 healthy controls.

\section{Methods}

\section{$\underline{\text { Study Population }}$}

The study was approved by the Emory Institutional Review Board, and all cases and controls provided written informed consent to participate. Subjects with a diagnosis of idiopathic CD were recruited from the Emory University Movement disorders clinic. Dystonia in other body regions was allowed (segmental and multifocal patterns), but involvement of neck muscles had to be the primary problem. Subjects were excluded if they had CD secondary to a known cause such as focal lesions, Parkinsonism or neuroleptic exposure. Control subjects were recruited from the same clinic. The majority of the controls were spouses or family members of the subjects with CD. Precise matching of cases and controls was not feasible.

We also collected information known to influence the metabolome. Subjects were asked if they use nicotine products currently or in the past or if they had a history of metabolic diseases (e.g., hypertension, hyperlipidemia, heart disease, or diabetes). Additionally, data about subjects' body mass index (BMI) and diet were also collected.

\section{$\underline{\text { Untargeted High-Resolution Metabolic Profiling }}$}

We conducted high-resolution metabolic profiling using liquid chromatography with ultra-high resolution mass spectrometry (LC-HRMS; Thermo Scientific Fusion) following established protocols[14] and published methods[15, 16]. Plasma specimens were collected and 
stored at $-80^{\circ} \mathrm{C}$ before thawing for liquid-chromatography mass spectrometry (LC/MS). Sam were treated with acetonitrile containing internal isotopic standard mix, centrifuged for 10 minutes at $4{ }^{\circ} \mathrm{C}$ to remove protein, and then maintained in a Dionex Ultimate 3000 autosampler at $4^{\circ} \mathrm{C}$ until analysis. NIST 1950 [17] was analyzed at the beginning and end of the entire analysis procedure. Two replicated pooled human plasma samples were analyzed at the beginning, middle, and end of each batch of 40 study samples for batch effect correction and normalization. Samples were analyzed in triplicate using C18 chromatography (Higgins endcapped C18 $2.1 \mathrm{~cm} \times 5 \mathrm{~cm} \times 3 \mu \mathrm{m}$ column) the following gradient program: initial $0.5 \mathrm{~min}$ period of $60 \% \mathrm{~A}$ (LCMS grade water), 35\% B (LCMS grade acetonitrile, 5\% C (10 mM ammonium acetate), followed by linear increase to $0 \% \mathrm{~A}, 95 \% \mathrm{~B}, 5 \% \mathrm{C}$ at $1.5 \mathrm{~min}$ which was held for an additional $3 \mathrm{~min}$. Finally, mobile phase flow rate was held at $0.4 \mathrm{~mL} / \mathrm{min}$ for $1.5 \mathrm{~min}$, and then increased to $0.5 \mathrm{~mL} / \mathrm{min}$. MS was operated in negative ionization mode using electrospray ionization, mass resolution of 120,000 and data collected from 85 to $1275 \mathrm{~m} / \mathrm{z}$ over 5 minutes)[15]. Source tune settings included capillary temperature, sheath gas, auxiliary gas, sweep gas and spray voltage settings of $300^{\circ} \mathrm{C}, 45$ (arbitrary units), 25 (arbitrary units), 1 (arbitrary units) and $+3.5 \mathrm{kV}$ for negative mode. S-Lens RF level was maintained at 45 . Feature extraction and alignment was performed in apLCMS [18], with quality control and feature filtering using xMSanalyzer [19]. Features are defined by their accurate mass $m / z$, retention time and ion abundance. Features with median coefficient of variation within technical replicates $\geq 75 \%$, and samples with Pearson correlation coefficient $\geq 0.7$ within triplicates were retained for downstream analysis. Batch effect was corrected using ComBat [20].

$\underline{\text { Feature Annotation and Identification }}$ 
Feature annotation was performed using xMSannotator [21] with the Human Metabolome Database [22]. Annotations with confidence score of 3 indicates high confidence level, score of 2 indicates medium confidence level. Only annotations with medium confidence or above were used. Furthermore, metabolite identification and confirmation was performed using an in-house library of metabolites that have been previously confirmed using comparison of retention time and MS/MS with authentic standards and comparison of MS/MS fragments with online spectral databases. The library was matched to the metabolic features that significantly associated with $\mathrm{CD}$, allowing an $\mathrm{m} / \mathrm{z}$ difference of $5 \mathrm{ppm}$ and a retention time difference of 20 seconds. Feature identification levels were assigned according to the published criteria [23]. At level 1, the proposed structure was confirmed via appropriate measurement of a reference standard with MS, MS/MS and retention time matching. At level 2, the proposed structure was confirmed by MS/MS and matched with online databases or in-silico predicted spectra. At level 3, the proposed structure was confirmed by MS/MS at the chemical class level, but no evidence for a specific metabolite. At level 4, the proposed structure was computationally annotated using xMSannotator with confidence level medium or above [21]. At level 5, the proposed structure was matched accurately by mass.

\section{$\underline{\text { Statistical Analysis }}$}

Subject characteristics were reported as mean (standard deviation) for continuous variables and frequency (percentage) for categorical variables. The differences between cases and controls were compared using two sample t-tests for continuous variables and Chi-square or Fisher's exact tests for categorical variables where appropriate. 
The metabolic feature intensities were summarized as median values of the non-zero readings across triplicates. Features were retained for analysis if there were less than $20 \%$ zero readings across $\mathrm{CD}$ samples and control samples. Intensities were $\log 2$ (intensity +1 ) transformed, mean centered and scaled by standard deviation.

Logistic regression model was utilized for the metabolome-wide association study (MWAS). The association was examined between the binary response variable of CD and each of the metabolic features, including age, gender, diet restrictions, and BMI as covariates. The Benjamini-Hochberg false discovery rate (FDR) [24] method was used to correct for multiple comparisons. Analyses were performed using R version 3.3.3 (R Foundation for Statistical Computing, Vienna, Austria) and SAS statistical software version 9.4 (SAS Institute Inc, Cary, NC).

\section{$\underline{\text { Pathway Analysis }}$}

Features associated with $\mathrm{CD}$ that had $\mathrm{p}<0.05$ in MWAS were chosen as target features that entered pathway analysis in Mummichog (version 2.1.1) [25], which is a software designed for high throughput untargeted metabolomics data. This analysis allows us to explore the functional activities directly from the untargeted features, bypassing metabolite identification. All features entered MWAS were utilized as the feature reference pool. Pathways enriched with $\mathrm{p}<0.05$ were considered as significant.

\section{Results}

Among the 200 subjects, 100 were CD cases and 100 were controls (Table 1). The proportion of females was significantly higher among cases than controls ( $78 \%$ vs $55 \%$, 
$\mathrm{p}<0.001)$. Cases were older compared to controls at enrollment $(62.3$ vs $57.5, p=0.011)$, and had lower BMI (26.1 vs 28.4, p=0.002). All of these variables were adjusted in the statistical model as covariates.

A total of 7,346 metabolic features passed quality control and entered MWAS. The MWAS revealed metabolomic associations for 289 features at $\mathrm{p}<0.05$, and 35 features at $\mathrm{p}<0.01$, and 1 feature at $\mathrm{p}<0.001$. None of these features were significant at FDR cutoff 0.2 (Figure $1 \&$ Supplemental Figure 1). Of the 289 features with $p<0.05,64$ features were annotated based on Human Metabolome Database (Supplemental Table 1). Table 2 presents the 6 annotated features out of the 35 with $p$ value $<0.01$. The 289 features associated with $C D$ at $p<0.05$ were further confirmed using the in-house library of confirmed metabolites. A total of 5 features were successfully matched (Table 3). Among those, higher level of uracil was associated with 36\% lower odds of CD (m/z 111.0199, time 13.4s, OR 0.64, 95\% CI 0.46-0.87, p=0.005).

Pathway analysis identified 9 significant pathways at $\mathrm{p}<0.05$ that are enriched based on the $289 \mathrm{CD}$-associated metabolic features, including prostaglandin formation from dihomo gama-linoleic acid $(\mathrm{p}<0.001)$, pentose phosphate pathway $(\mathrm{p}=0.005)$, arachidonic acid metabolism $(\mathrm{p}=0.013)$, leukotriene metabolism $(\mathrm{p}=0.015)$, starch and sucrose metabolism $(\mathrm{p}=0.030)$, valine, leucine and isoleucine degradation $(\mathrm{p}=0.031)$, galactose metabolism $(p=0.031)$, fructose and mannose metabolism $(p=0.047)$ and propanoate metabolism $(p=0.047)$ (Table 4).

\section{Discussion}

This report describes the first large-scale metabolomics study for CD, the most common form of the idiopathic adult-onset dystonia. A total of 7,346 metabolites were measured, and 
significant differences could be detected between $\mathrm{CD}$ and control samples for individual metabolites. Pathway analysis revealed 9 biological processes to be associated at $\mathrm{p}<0.05$. These results provide novel insights into the biological basis for $\mathrm{CD}$.

An interesting metabolite that was identified as potentially abnormal in CD was the oxidized lipid 2-hydroxydecanoate. This metabolite was also identified in patients with Parkinson disease associated with mutations in parkin [26]. Dystonia is a well-known feature of this genetic subtype of Parkinson disease, although the dystonia is mostly in the limbs [27-29]. Docosahexaenoic acid was also potentially abnormal in CD. This metabolite is an omega-3 fatty acid which is essential for brain development and normal function, and many studies have implied that dystonia is a disorder of neural development or maladaptive plasticity [30, 31].

Among the biological pathways that were significantly associated with CD (Table 4), five were related to carbohydrate metabolism, suggesting a potential link with energy production. Three pathways were related to lipid metabolism, which produces prostaglandins and other metabolites known to mediate inflammatory responses [32, 33]. Involvement of these pathways may suggest a link to an immune-related process in dystonia. Several case series have reported CD to be associated with autoimmune diseases such as systemic lupus erythematosus, myasthenia gravis and Sjogrens syndrome [34-37]. CD can also occur as a delayed consequence of various infections, due to immune system cross-reactivity between the infectious agent and host targets [38-40]. Thus, the immune link suggested by our metabolomics findings may be worthy of further investigation.

Although this study has revealed several intriguing novel potential biological leads for future studies, the results must be interpreted with caution. Numerous variables are known to affect the metabolome in human blood samples, and it is not feasible to control for all of them. 
For instance, treatments for CD such as botulinum toxin injections may affect the metabolome. The timing of these injections is difficult to be incorporated in the statistical model due to missing data, and the dosing pattern varies individually. Although this study was based on a relatively large number of cases and controls all collected in a standardized manner at a single center, a replication study is still warranted. Because of the large number of measurements made, correction for multiple comparisons is needed to increase confidence in the findings. On the other hand, because this study was exploratory, the application of overly conservative statistical criteria is not helpful either, because important novel leads may be inappropriately overlooked. We therefore chose to report findings at different statistical thresholds. Additionally, due to the cross-sectional study design, no temporality between the different metabolomic profiles and CD can be inferred. A longitudinal cohort study with follow-up may be needed to better understand the disease onset and progression. Finally, using blood samples to infer biological mechanisms for a brain disorder carries significant limitations, given that relevant biomarkers may not cross the blood-brain barrier. Further investigations of cerebral spinal fluid or brain samples may be needed in future studies. 


\section{Acknowledgements}

This work was supported by the National Institute for Neurological Disorders and Stroke (NS096455 and NS116025) and the Rare Diseases Clinical Research Network of the Office of Rare Diseases Research at the National Center for Advancing Translational Sciences (TR001456).

\section{Author's Roles}

YVS and HAJ were involved in the conception, organization and execution of the research project; , LS, GK, AC, SF, AF and HAJ contributed to evaluation of cases, and phenotypic data collection and biospecimen collection; KU and DJ were responsible for the generation of metabolomic data; CL, KU and YVS were responsible of the design and execution of statistical analysis; The first draft was written by CL, HAJ and YVS, and developed further by LS, and GK. All co-authors contributed to critical review and editing of the manuscript.

\section{Financial Disclosures}

H. A. Jinnah has active or recent grant support from the US government (National Institutes of Health), private philanthropic organizations (the Benign Essential Blepharospasm Research Foundation, Cure Dystonia Now), academically-oriented institutions (the Dystonia Study Group), and industry (Cavion Therapeutics, Ipsen Pharmaceuticals, Retrophin Inc.). Dr. Jinnah has also served on advisory boards or as a consultant for Abide Therapeutics, Allergan Inc., CoA Therapeutics, and Retrophin Inc. He has received honoraria or stipens for lectures or administrative work from the American Academy of Neurology, the American Neurological Association, the Dystonia Medical Research Foundation, the International Neurotoxin Society, 
the International Parkinson's Disease and Movement Disorders Society, The Parkinson's Disease Foundation, and Tyler's Hope for a Cure. Dr. Jinnah serves on the Scientific Advisory Boards for several private foundations including the Benign Essential Blepharospasm Research Foundation, Cure Dystonia Now, the Dystonia Medical Research Foundation, the Tourette Association of American, and Tyler's Hope for a Cure. He also is principle investigator for the Dystonia Coalition, which receives the majority of its support through NIH grants NS116025 and NS065701 from the National Institutes of Neurological Disorders and Stroke and TR001456 from the Office of Rare Diseases Research at the National Center for Advancing Translational Sciences. The Dystonia Coalition has received additional material or administrative support from industry sponsors (Allergan Inc. and Merz Pharmaceuticals) as well as private foundations (The American Dystonia Society, Beat Dystonia, The Benign Essential Blepharospasm Foundation, Cure Dystonia Now, Dystonia Europe, Dystonia Inc., Dystonia Ireland, The Dystonia Medical Research Foundation, The Foundation for Dystonia Research, The National Spasmodic Dysphonia Association, and The National Spasmodic Torticollis Association).

Dr. Factor has the following disclosures: Honoraria: Lundbeck, Teva, Sunovion, Biogen, Acadia, Neuroderm, Acorda, CereSpir. Grants: Ipsen, Medtronics, Boston Scientific, Teva, US World Meds, Sunovion Therapeutics, Vaccinex, Voyager, Jazz Pharmaceuticals, Lilly, CHDI Foundation, Michael J. Fox Foundation, NIH (U10 NS077366). Royalties: Demos, Blackwell Futura, Springer for textbooks, Uptodate and Other Bracket Global LLC, CNS Ratings LL 
Table 1. Baseline characteristics.

\begin{tabular}{|c|c|c|c|c|}
\hline Variable & Level & Control $N=100$ & $C D N=100$ & P-value* \\
\hline Female $(\%)$ & & $55(55)$ & $78(78)$ & $<.001$ \\
\hline \multirow[t]{3}{*}{ Ethnicity } & Black $(\%)$ & $10(10)$ & $4(4)$ & 0.179 \\
\hline & White $(\%)$ & $88(88)$ & $95(95)$ & \\
\hline & Other $(\%)$ & $2(2)$ & $1(1)$ & \\
\hline Age in year (sd) & & $57.5(14.6)$ & $62.3(11.9)$ & 0.011 \\
\hline BMI in $\mathrm{kg} / \mathrm{m}^{2}(\mathrm{sd})$ & & $28.4(6.0)$ & $26.1(4.4)$ & 0.002 \\
\hline Weight in $\mathrm{lb}$ (sd) & & $181.4(48.4)$ & $162.2(34.7)$ & 0.001 \\
\hline Height in inch (sd) & & $67.1(4.0)$ & $66.2(3.8)$ & 0.074 \\
\hline Dietary restrictions $(\%)$ & & $9(9)$ & $15(15)$ & 0.277 \\
\hline \multirow[t]{3}{*}{ Smoking } & Current smoker (\%) & $7(7)$ & $7(7)$ & 0.912 \\
\hline & Past smoker (\%) & $27(27)$ & $30(30)$ & \\
\hline & Never smoker $(\%)$ & $64(64)$ & $62(62)$ & \\
\hline
\end{tabular}

*Two-sample t-tests used for continuous variables; Chi-square tests or Fisher's exact tests used for categorical variables where appropriate. 
Table 2. Metabolic features matched to possible annotations significantly associated with CD in MWAS at $\mathbf{p}<0.01$.

\begin{tabular}{|c|c|c|c|c|c|c|c|c|c|}
\hline $\mathbf{m} / \mathbf{z}$ & Time(s) & $\begin{array}{l}\text { Odds } \\
\text { Ratio } \\
\end{array}$ & $95 \% \mathrm{CI}$ & P-value & FDR & Confidence & Potential Matching Annotations & HMDB $^{*}$ ID & Adduct \\
\hline 194.0462 & 19.5 & 0.53 & $0.37,0.77$ & 0.001 & 0.904 & 2 & $\begin{array}{c}\text { Salicyluric acid; } \\
\text { Dopaquinone; } \\
\text { 4-Carboxyphenylglycine; } \\
\text { Alpha-Hydroxyhippuric acid; } \\
\text { Leucodopachrome; } \\
\text { 3-Hydroxyhippuric acid; } \\
\text { 4-Hydroxyhippuric acid; } \\
\text { 2-Hydroxy-67- } \\
\text { dimethoxybenzoxazole; } \\
\text { 2-Hydroxy-7-methoxy-2H-14- } \\
\text { benzoxazin-3(4H)-one; } \\
\text { L-Dopaquinone; } \\
\text { N-acetyl-5-aminosalicylic acid }\end{array}$ & $\begin{array}{l}\text { HMDB00840; } \\
\text { HMDB01229; } \\
\text { HMDB02016; } \\
\text { HMDB02404; } \\
\text { HMDB04067; } \\
\text { HMDB06116; } \\
\text { HMDB13678; } \\
\text { HMDB30726; } \\
\text { HMDB38055; } \\
\text { HMDB39119; }\end{array}$ & M-H \\
\hline 551.3594 & 205.3 & 0.56 & $0.40,0.80$ & 0.002 & 0.904 & 2 & Cyclopassifloic acid E & HMDB36298 & M-H \\
\hline 160.0252 & 16.9 & 0.60 & $0.43,0.84$ & 0.003 & 0.904 & 2 & $\begin{array}{l}\text { 4-Hydroxy-L-glutamic acid; } \\
\text { M- Ketoglutaric acid oxime; } \\
\text { Erucin; } \\
\text { M- Thiocyanato-4- } \\
\text { (methylthio)butane; } \\
\text { N-Formyl-L-aspartate }\end{array}$ & $\begin{array}{l}\text { HMDB02273; } \\
\text { HMDB02467; } \\
\text { HMDB33764; } \\
\text { HMDB38446; } \\
\text { HMDB60495 }\end{array}$ & $\mathrm{M}-\mathrm{H}$ \\
\hline 111.0199 & 13.4 & 0.633 & $0.46,0.87$ & 0.005 & 0.904 & 2 & $\begin{array}{c}\text { Uracil; } \\
\text { 4-Carboxypyrazole }\end{array}$ & $\begin{array}{l}\text { HMDB00300; } \\
\text { HMDB60760 }\end{array}$ & $\mathrm{M}-\mathrm{H}$ \\
\hline 287.0716 & 186.2 & 0.63 & $0.45,0.88$ & 0.008 & 0.904 & 2 & $\begin{array}{l}\text { Hydroxyanigorufone; } \\
\text { Irenolone }\end{array}$ & $\begin{array}{l}\text { HMDB34449; } \\
\text { HMDB40716 }\end{array}$ & $\mathrm{M}-\mathrm{H}$ \\
\hline 237.1859 & 177.2 & 1.70 & $1.13,2.53$ & 0.010 & 0.904 & 2 & $\begin{array}{l}\text { Geranyl 3-methylbutanoate; } \\
\text { Linalyl isovalerate; }\end{array}$ & $\begin{array}{l}\text { HMDB29350; } \\
\text { HMDB30428; } \\
\text { HMDB31383; }\end{array}$ & M-H \\
\hline
\end{tabular}




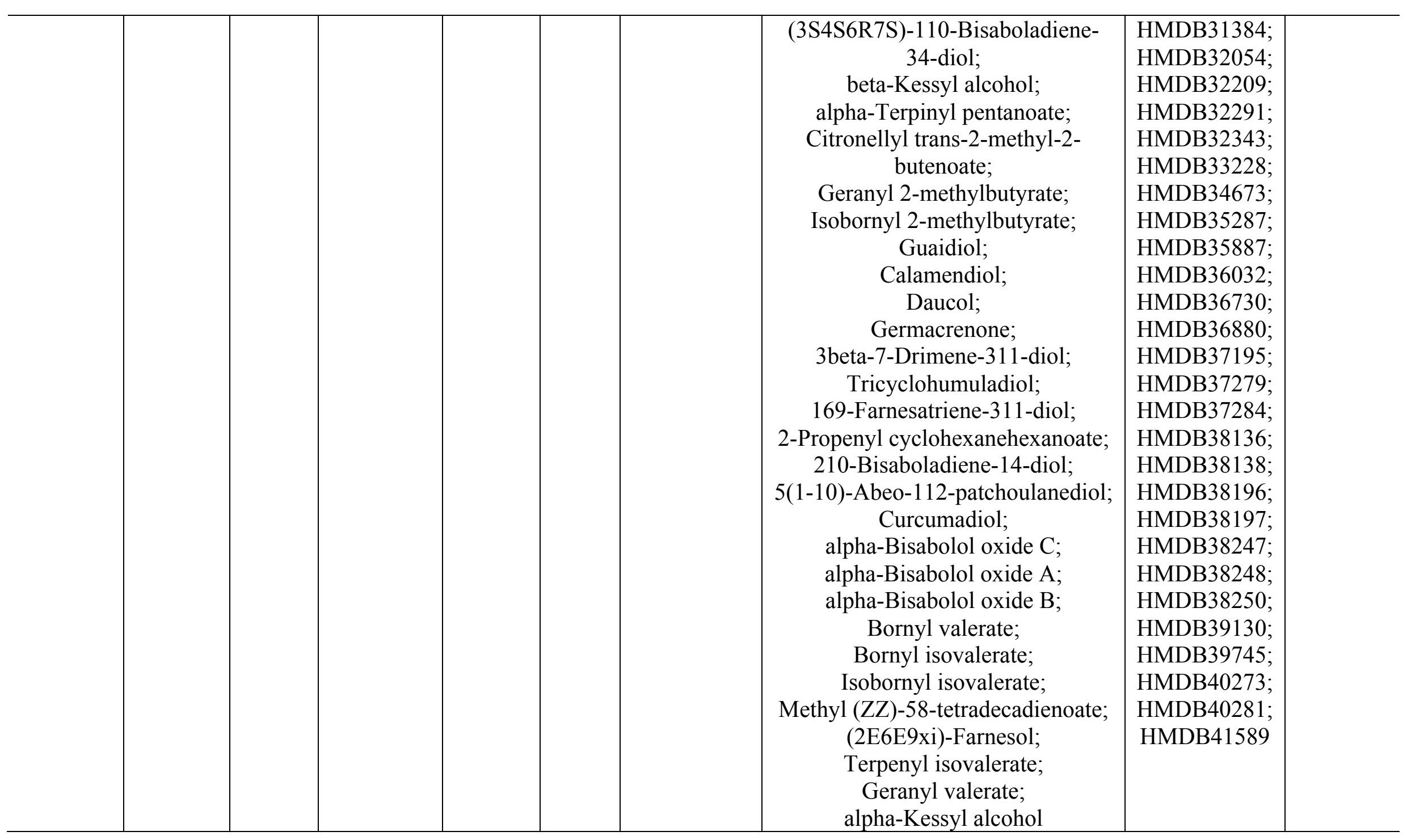

* HMDB: Human Metabolome Database 
Table 3. Confirmed features significantly associated with CD in MWAS at $\mathbf{p}<0.05$.

\begin{tabular}{c|c|c|c|c|c|c|c}
\hline $\mathbf{m} / \mathbf{z}$ & Time(s) & Odds Ratio & $\mathbf{9 5 \%}$ CI & P-value & Chemical Name & KEGG* ID & HMDB** ID \\
\hline 111.0199 & 13.4 & 0.64 & $0.46,0.87$ & 0.005 & Uracil & C00106 & HMDB00300 \\
\hline 187.1338 & 22.1 & 1.43 & $1.05,1.94$ & 0.024 & 10 -Hydroxydecanoate & C02774 & \\
\hline 177.0407 & 19 & 1.41 & $1.02,1.93$ & 0.036 & D-Gulonic Acid Gamma-Lactone & C01040 & HMDB03466 \\
\hline 327.2333 & 234.9 & 1.40 & $1.02,1.91$ & 0.036 & Docosahexaenoic Acid & C06429 & HMDB02183 \\
\hline 89.0244 & 24.4 & 1.37 & $1.02,1.86$ & 0.038 & (S)-Lactate; Glyceraldehyde & $\begin{array}{c}\text { C01432; } \\
\text { C02154 }\end{array}$ & $\begin{array}{c}\text { HMDB000190; } \\
\text { HMDB01051 }\end{array}$ \\
\hline 173.0569 & 17.9 & 0.73 & $0.54,0.99$ & 0.046 & N-Alpha-Acetyl-L-Asparagine & CA1214 & HMDB06028 \\
\hline
\end{tabular}

*KEGG: Kyoto Encyclopedia of Genes and Genomes

**HMDB: Human Metabolome Database 
Table 4. Significant pathways based on the 289 features associated with CD at $p<0.05$ in MWAS.

\begin{tabular}{|c|c|c|c|}
\hline Pathways & Overlap size & Pathway size & P-value \\
\hline Prostaglandin formation from dihomo gama-linoleic acid & 3 & 5 & $<.001$ \\
\hline Pentose phosphate pathway & 4 & 22 & 0.005 \\
\hline Arachidonic acid metabolism & 3 & 17 & 0.013 \\
\hline Leukotriene metabolism & 3 & 18 & 0.015 \\
\hline Starch and Sucrose Metabolism & 2 & 10 & 0.030 \\
\hline Valine, leucine and isoleucine degradation & 3 & 23 & 0.031 \\
\hline Galactose metabolism & 3 & 23 & 0.031 \\
\hline Fructose and mannose metabolism & 2 & 14 & 0.047 \\
\hline Propanoate metabolism & 2 & 14 & 0.047 \\
\hline
\end{tabular}


Figure 1. Manhattan plot of the metabolome-wide association study of CD. Total of 7,346 features. Dashed line indicates $\mathrm{p}=0.05 .157$ features with $\mathrm{p}<0.05$ and $\mathrm{OR}<1$ colored in red; 132 features with $\mathrm{p}<0.05$ and $\mathrm{OR}>1$ colored in blue.

A.

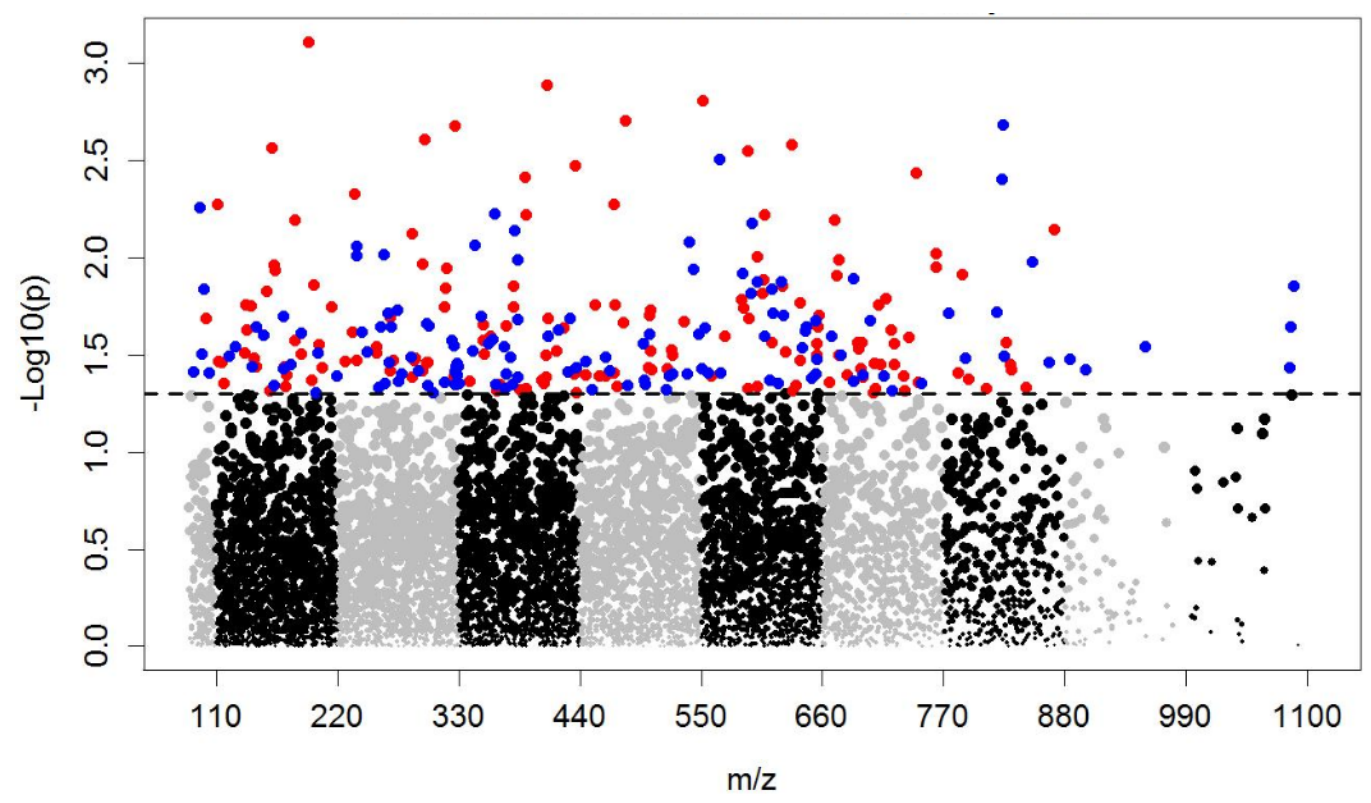

B.

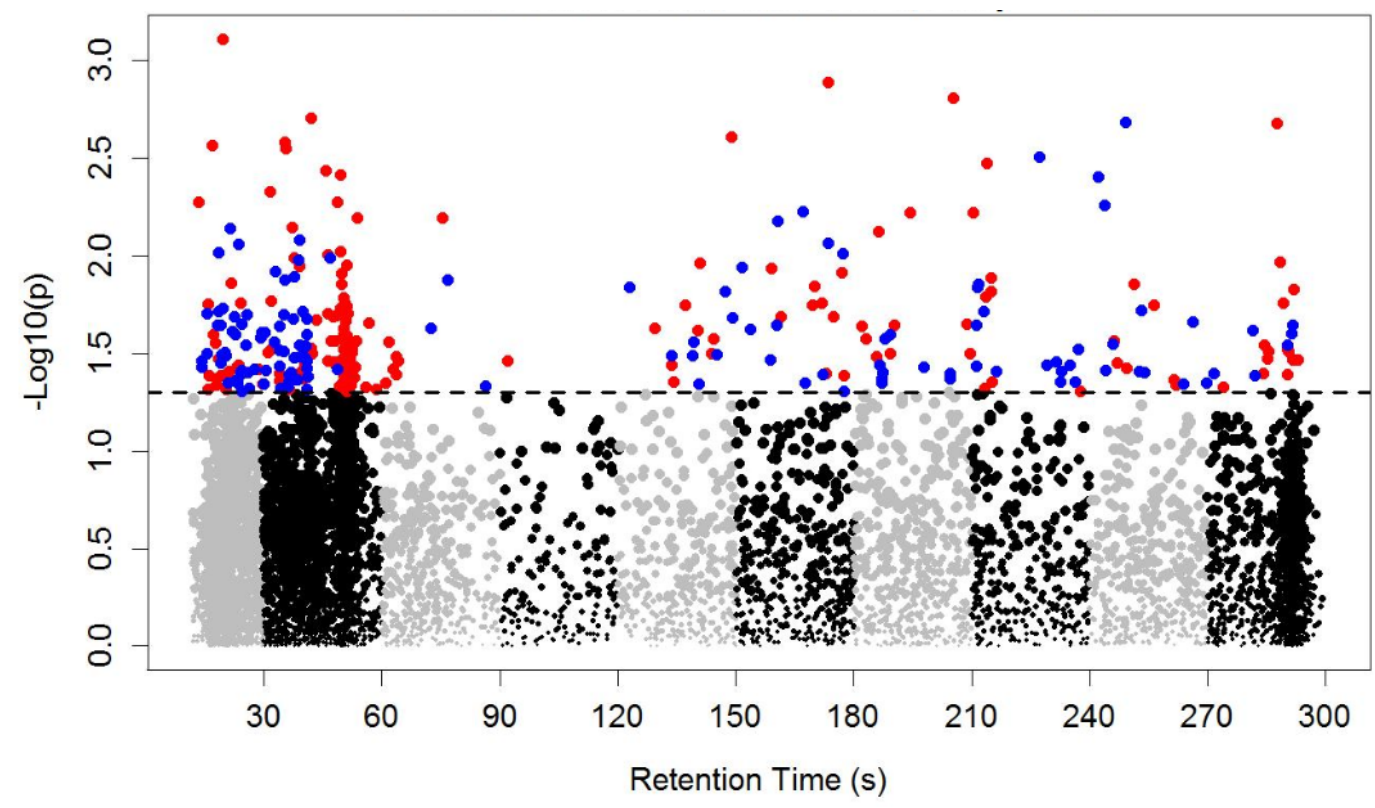


Figure 2. Volcano plot of the metabolome-wide association study of CD. Total of 7,346 features. Horizontal dashed line indicates $\mathrm{p}=0.05$. 157 features with $\mathrm{p}<0.05$ and $\mathrm{OR}<1$ colored in red; 132 features with $\mathrm{p}<0.05$ and $\mathrm{OR}>1$ colored in blue.

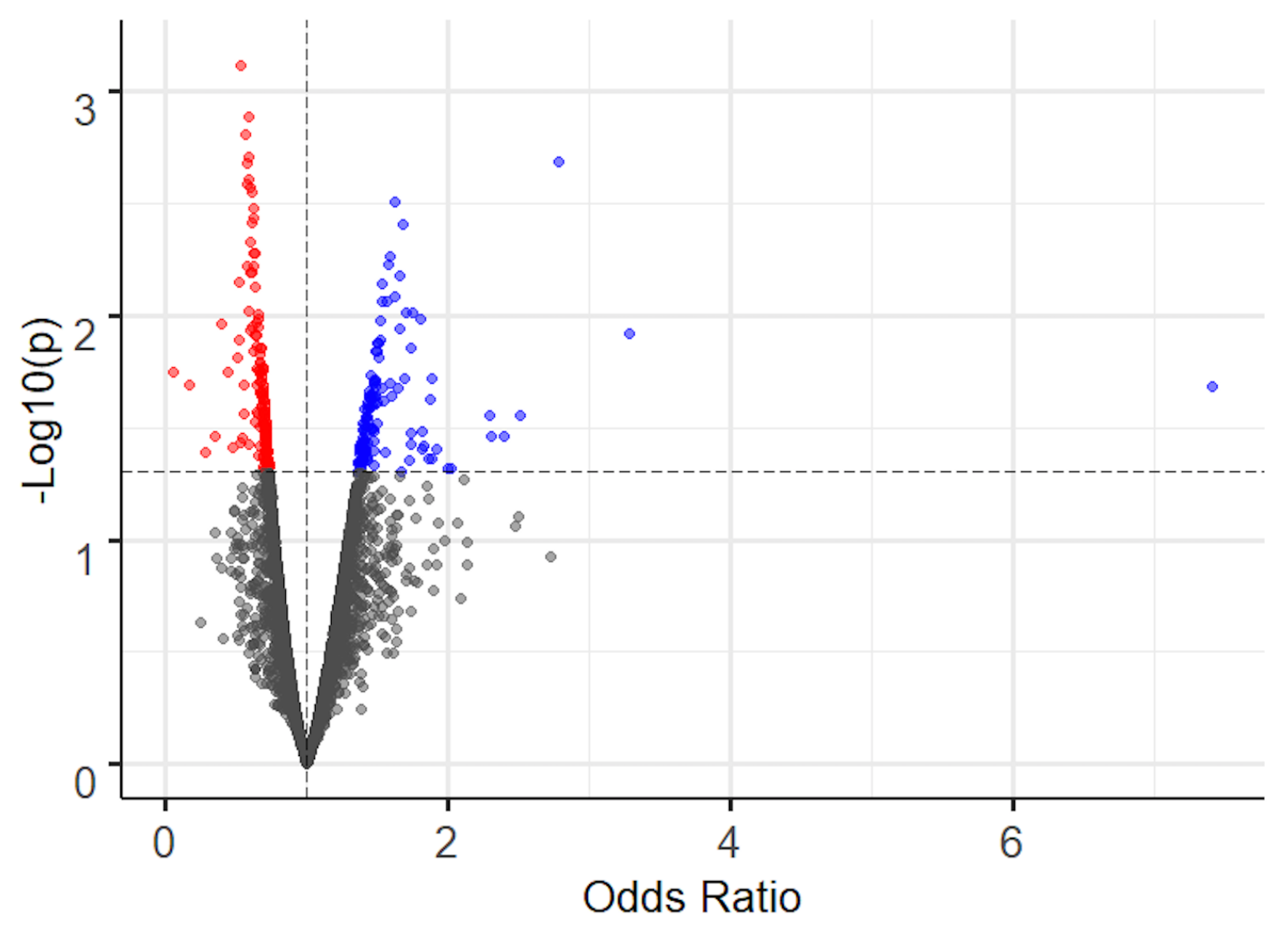




\section{Reference}

1. LeDoux, M.S., et al., Clinical and genetic features of cervical dystonia in a large multicenter cohort. Neurol Genet, 2016. 2(3): p. e69.

2. Claypool, D.W., et al., Epidemiology and outcome of cervical dystonia (spasmodic torticollis) in Rochester, Minnesota. Mov Disord, 1995. 10(5): p. 608-14.

3. Nutt, J.G., et al., Epidemiology of focal and generalized dystonia in Rochester, Minnesota. Mov Disord, 1988. 3(3): p. 188-94.

4. Jinnah, H.A., et al., The focal dystonias: current views and challenges for future research. Mov Disord, 2013. 28(7): p. 926-43.

5. Corp, D.T., et al., Network localization of cervical dystonia based on causal brain lesions. Brain, 2019. 142(6): p. 1660-1674.

6. Factor, S.A., et al., Recent developments in drug-induced movement disorders: a mixed picture. Lancet Neurol, 2019. 18(9): p. 880-890.

7. Savitt, D. and J. Jankovic, Tardive syndromes. J Neurol Sci, 2018. 389: p. 35-42.

8. Bredenkamp, J.K. and D.R. Maceri, Inflammatory torticollis in children. Arch Otolaryngol Head Neck Surg, 1990. 116(3): p. 310-3.

9. Balint, B., et al., Movement disorders with neuronal antibodies: syndromic approach, genetic parallels and pathophysiology. Brain, 2018. 141(1): p. 13-36.

10. Newman, J.R., et al., Risk factors for idiopathic dystonia in Queensland, Australia. J Clin Neurosci, 2014. 21(12): p. 2145-9.

11. Molloy, A., et al., An evaluation of the role of environmental factors in the disease penetrance of cervical dystonia. J Neurol Neurosurg Psychiatry, 2015. 86(3): p. 331-5.

12. Jones, D.P., Y. Park, and T.R. Ziegler, Nutritional metabolomics: progress in addressing complexity in diet and health. Annu Rev Nutr, 2012. 32: p. 183-202.

13. Patti, G.J., O. Yanes, and G. Siuzdak, Innovation: Metabolomics: the apogee of the omics trilogy. Nat Rev Mol Cell Biol, 2012. 13(4): p. 263-9.

14. Go, Y.M., et al., Reference Standardization for Mass Spectrometry and High-resolution Metabolomics Applications to Exposome Research. Toxicol Sci, 2015. 148(2): p. 531-43.

15. Walker, D.I., et al., Metabolomic assessment of exposure to near-highway ultrafine particles. J Expo Sci Environ Epidemiol, 2019. 29(4): p. 469-483.

16. Walker, D.I., et al., Metabolome-wide association study of anti-epileptic drug treatment during pregnancy. Toxicol Appl Pharmacol, 2019. 363: p. 122-130. 
17. Simon-Manso, Y., et al., Metabolite profiling of a NIST Standard Reference Material for human plasma (SRM 1950): GC-MS, LC-MS, NMR, and clinical laboratory analyses, libraries, and web-based resources. Anal Chem, 2013. 85(24): p. 11725-31.

18. Yu, T., et al., apLCMS--adaptive processing of high-resolution LC/MS data. Bioinformatics, 2009. 25(15): p. 1930-6.

19. Uppal, K., et al., xMSanalyzer: automated pipeline for improved feature detection and downstream analysis of large-scale, non-targeted metabolomics data. BMC Bioinformatics, 2013. 14: p. 15.

20. Johnson, W.E., C. Li, and A. Rabinovic, Adjusting batch effects in microarray expression data using empirical Bayes methods. Biostatistics, 2007. 8(1): p. 118-27.

21. Uppal, K., D.I. Walker, and D.P. Jones, xMSannotator: An R Package for Network-Based Annotation of High-Resolution Metabolomics Data. Anal Chem, 2017. 89(2): p. 10631067.

22. Wishart, D.S., et al., HMDB: the Human Metabolome Database. Nucleic Acids Res, 2007. 35(Database issue): p. D521-6.

23. Schymanski, E.L., et al., Identifying small molecules via high resolution mass spectrometry: communicating confidence. Environ Sci Technol, 2014. 48(4): p. 2097-8.

24. Benjamini, Y. and Y. Hochberg, Controlling the False Discovery Rate: A Practical and Powerful Approach to Multiple Testing. Journal of the Royal Statistical Society. Series B (Methodological), 1995. 57(1): p. 289-300.

25. Li, S., et al., Predicting network activity from high throughput metabolomics. PLoS Comput Biol, 2013. 9(7): p. e1003123.

26. Okuzumi, A., et al., Metabolomics-based identification of metabolic alterations in PARK2. Ann Clin Transl Neurol, 2019. 6(3): p. 525-536.

27. de Schipper, L.J., A.J.W. Boon, and A.G. Munts, Foot Drop Dystonia Resulting from parkin (PARK2) Mutation. Mov Disord Clin Pract, 2015. 2(3): p. 292-294.

28. Potulska-Chromik, A., et al., Dopa-responsive dystonia or early-onset Parkinson disease - Genotype-phenotype correlation. Neurol Neurochir Pol, 2017. 51(1): p. 1-6.

29. Benger, M., et al., The Interaction of Genetic Mutations in PARK2 and FA2H Causes a Novel Phenotype in a Case of Childhood-Onset Movement Disorder. Front Neurol, 2019. 10: p. 555.

30. Quartarone, A. and A. Pisani, Abnormal plasticity in dystonia: Disruption of synaptic homeostasis. Neurobiol Dis, 2011. 42(2): p. 162-70. 
31. Quartarone, A. and M. Hallett, Emerging concepts in the physiological basis of dystonia. Mov Disord, 2013. 28(7): p. 958-67.

32. Wang, X., H. Lin, and Y. Gu, Multiple roles of dihomo-gamma-linolenic acid against proliferation diseases. Lipids Health Dis, 2012. 11: p. 25.

33. Hanna, V.S. and E.A.A. Hafez, Synopsis of arachidonic acid metabolism: A review. J Adv Res, 2018. 11: p. 23-32.

34. Renganathan, R., et al., Sustained dystonia as the initial presentation of multiple sclerosis. Ir Med J, 2005. 98(7): p. 219-20.

35. Rajagopalan, N., P.R. Humphrey, and R.C. Bucknall, Torticollis and blepharospasm in systemic lupus erythematosus. Mov Disord, 1989. 4(4): p. 345-8.

36. van den Berg, J.S., et al., Dystonia; a central nervous system presentation of Sjogren's syndrome. Mov Disord, 1999. 14(2): p. 374-5.

37. Hinduja, A., et al., Dystonia with superimposed myasthenia gravis: An experiment in nature. Mov Disord, 2008. 23(11): p. 1626-7.

38. Green, C. and D.E. Riley, Treatment of dystonia in striatal necrosis caused by Mycoplasma pneumoniae. Pediatr Neurol, 2002. 26(4): p. 318-20.

39. Dale, R.C., et al., Post-streptococcal autoimmune dystonia with isolated bilateral striatal necrosis. Dev Med Child Neurol, 2002. 44(7): p. 485-9.

40. Edwards, M.J., et al., A dystonic syndrome associated with anti-basal ganglia antibodies. J Neurol Neurosurg Psychiatry, 2004. 75(6): p. 914-6. 